\title{
Ablation efficiency of $\alpha-\mathrm{Al}_{2} \mathrm{O}_{3}$ in liquid phase and ambient air by nanosecond laser irradiation
}

\author{
Csaba László Sajti · Ramin Sattari • Boris Chichkov • \\ Stephan Barcikowski
}

Received: 21 September 2009 / Accepted: 6 January 2010 / Published online: 11 February 2010

(C) The Author(s) 2010. This article is published with open access at Springerlink.com

\begin{abstract}
Ablation efficiency and influences of laser parameters on a material removal rate by a nanosecond laser irradiation of $\alpha-\mathrm{Al}_{2} \mathrm{O}_{3}$ are studied in gas and liquid phases. The laser ablation in the air yields maximum material removal rate of $12 \mathrm{ng} /$ pulse using a 4.6- $\mathrm{mJ}$ pulse energy at $4-\mathrm{kHz}$ repetition rate, compared to $88 \mathrm{ng} /$ pulse in the water flow. Using a specific interpulse distance and a laser repetition rate further increase material removal rate by factor of 3 and 65 , respectively, owing to an optimized lattice temperature and laser pulse interactions with the generated cavitation bubble. For the ablation in the air, these parameters do not significantly affect the ablation efficiency.
\end{abstract}

\section{Introduction}

During the last decade the laser ablation of solid substrates was widely investigated in a vacuum and various gas environments, targeting diverse applications including cutting, drilling, welding, or thin-film deposition, but comparatively few studies have been reported on a laser ablation using a solid-liquid interface [1-3]. Nevertheless, laser ablation in liquids attracts more and more attention enabling the generation of nanoparticle colloids with outstanding purity compared to the conventional manufacturing routes [4]. Recently, it has been shown that the laser ablation in a liquid media may initiate a higher ablation rate than that observed in a gas phase [5]. It has been assumed that the ablation in a liquid generates confined plasma with higher pressure [6]

C.L. Sajti · R. Sattari · B. Chichkov · S. Barcikowski $(\bowtie)$ Laser Zentrum Hannover e.V., Hollerithallee 8, 30419 Hannover, Germany

e-mail: s.barcikowski@lzh.de

Fax: +49-511-2788100 than that in ambient air, yielding the enhancement in the ablation rate and mechanical responses of substrates (e.g., shockwave lasts three times longer when ablating in a liquid). Another study investigated the thickness of the utilized liquid layer and assumed an optimal plasma and shockwave pressure in a specific liquid height [7]. In this high pressure reaction field, during laser ablation in liquids, a cavitation bubble forms which expands, shrinks, and collapses with time $[8,9]$ and is presumed to affect material removal rate. However, the main parameters and mechanisms contributing to the enhanced ablation efficiency during a laser material processing in liquids are still subject to further investigations.

In this letter a series of experiments is reported to compare laser ablation efficiencies of solid $\alpha-\mathrm{Al}_{2} \mathrm{O}_{3}$ in a waterconfined media and in an ambient air. The ablation rate is measured and compared at various laser fluences, repetition rates, and interpulse distances. In a liquid environment primary particle size, size distribution, and particle morphology are also investigated.

\section{Experimental details}

The laser ablation was carried out at room temperature at atmospheric pressure in an ambient air or in a distilledwater media with a commercial laser system (Rofin Sinar Marker 100D) providing 40 to $55 \mathrm{~ns}$ full width at half maximum (FWHM) laser pulses at $1064 \mathrm{~nm}$ (maximum pulse energy $8 \mathrm{~mJ}$, repetition rate 0.5 to $20 \mathrm{kHz}$ ). The irradiated 600- $\mu \mathrm{m}$ thick corundum target (Deranox 975, Morgan Technical Ceramics) was prepared by a hot isostatic pressing of alpha $\mathrm{Al}_{2} \mathrm{O}_{3}$ powder with purity of $97.5 \%$ and grain size of five microns. The irradiation was delivered by 
a laser scanner (Scanlab, HurryScan II-14) through a telecentric 58-mm focal length F-theta lens (Sill Optics). The applied scan speed varied from 10 to $1400 \mathrm{~mm} / \mathrm{s}$ over a fixed $8 \mathrm{~mm}$ by $8 \mathrm{~mm}$ multiline ablation pattern providing $50 \mu \mathrm{m}$ of interline distance. In a liquid environment the focused beam was coupled horizontally into a process chamber filled with $65 \mathrm{~mL}$ of distilled water through an antireflexcoated sapphire window. The controlled liquid flow on the target surface was generated by a flexible tube pump being $190 \mathrm{~mL} / \mathrm{min}$ throughout the present study, as in the fluence regime investigated this special liquid flow allowed the efficient removal of processed gas bubbles from the ablation zone and resulted in the rapid dispersion of nanoparticles into the whole liquid volume. The utilized flow chamber yields a stable and highly reproducible ablation process with a variation in absolute material mass removal values of less than $3 \%$ as the ablation process is less affected by the formation of vapor bubbles and the thermal distortion of the laser beam [10]. In this study 5 min of irradiation time was chosen after ensuring that no significant discrepancy from linearity is involved, comparing the amount of ablated material after $5,10,15$, and 30 min of ablation.

\section{Results and discussion}

Figure 1a demonstrates the influence of a laser pulse energy on $\alpha-\mathrm{Al}_{2} \mathrm{O}_{3}$ material removal rates in an ambient air and using 6-mm-thick water layer at $120 \mathrm{~mm} / \mathrm{s}$ scan speed and a laser focal spot of $80 \mu \mathrm{m}$ in diameter. In liquid flow the redeposition of an ablated matter is considerably reduced, resulting in a great enhancement in the ablation rate of alumina [5]. Using $3.9 \mathrm{~mJ}$ of focused laser pulse energy with $5-\mathrm{kHz}$ repetition rate, 7 and $25 \mathrm{ng} /$ pulse of material removal rates were achieved in an air and water, respectively. In the range of 0.7 to $3 \mathrm{~mJ}$ ( 14 to $50 \mathrm{~J} / \mathrm{cm}^{2}$ of laser fluence), nanoparticle productivity increased about linearly (with different slopes in an air and water) with pulse energy. Exciting nonlinear phenomena seem to appear in both environments when a pulse energy exceeds $3.5 \mathrm{~mJ}$ (laser fluence of $70 \mathrm{~J} / \mathrm{cm}^{2}$ ). This fact proves that a promoted ablation rate in this fluence regime is driven only by light-material interaction and not environmental effects. Calculation of ablation efficiencies from Fig. 1a clearly reveals several ablation regimes and completely different ablation processes in an ambient air and water, presented in Fig. 1b. In water, increasing laser fluence leads to abrupt enhancement in the ablation efficiency followed by a saturation domain in the low-middle fluence regime where we presume that normal vaporization is the major material removal mechanism [11, 12]. Extrapolation of the linear part of the curve denotes the threshold fluence of $\mathrm{Al}_{2} \mathrm{O}_{3}$ of about $14.5 \mathrm{~J} / \mathrm{cm}^{2}$. The saturation plateau discloses slight decrease in the ablation efficiency with increasing laser fluence, revealing that absorption and scattering of
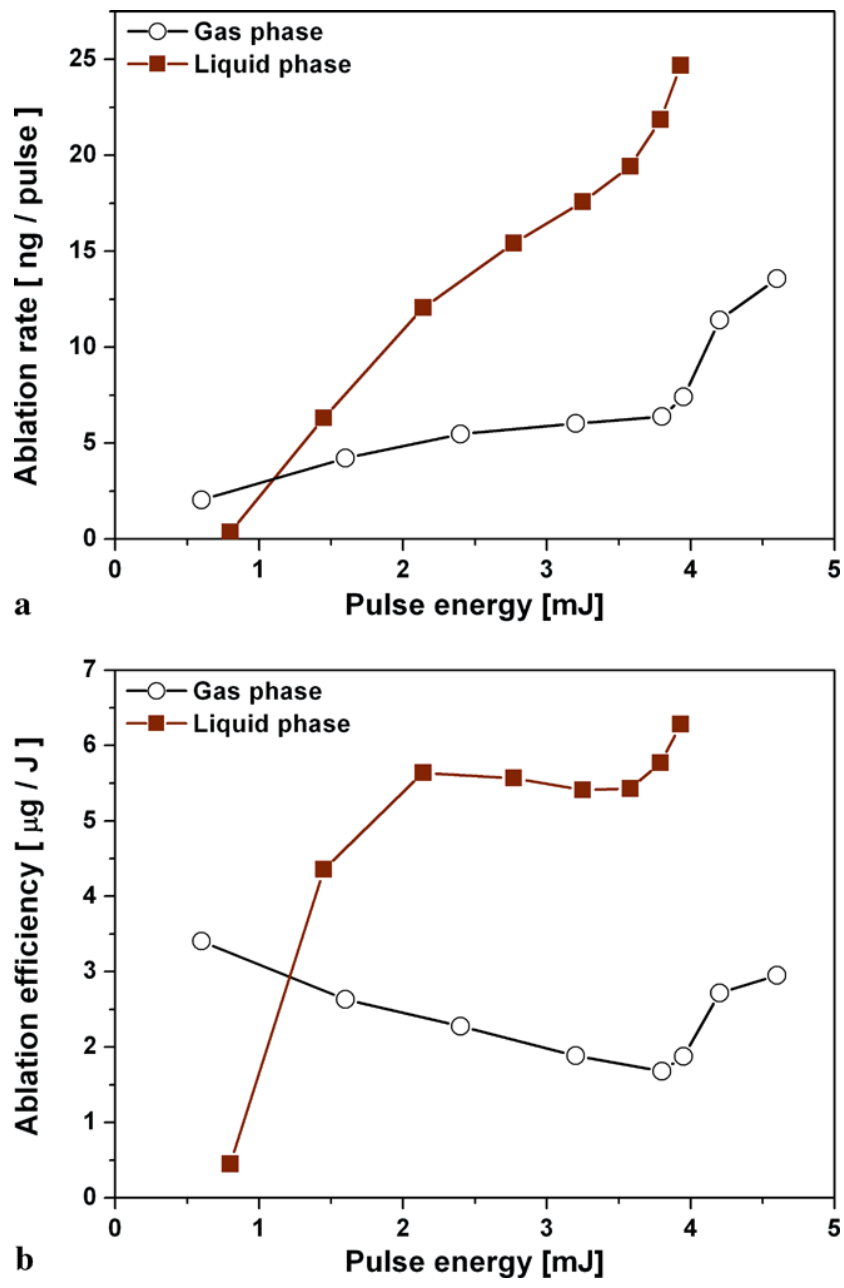

Fig. 1 (a) $\mathrm{Al}_{2} \mathrm{O}_{3}$ ablation rate as a function of the laser pulse energy in an ambient air and in water using 5-kHz repetition rate. (b) Ablation efficiencies as a function of laser pulse energy

the laser beam on previously ablated nanoparticles become significant at high fluences in water. Ablating in an ambient air with increasing pulse energy yields continuous reduction in the ablation efficiencies, indicating that the most probable debris redeposition is linearly dependent on a laser fluence. Exceeding $3.5 \mathrm{~mJ}$ laser pulse energy, roughly quadratic raise of the ablation efficiency in an air and water indicates the change in ablation mechanism. Phase explosion may be the responsible mechanism for such violent increase in laser ablation efficiency [12]. However, further investigations are necessary for better understanding of such increase in the high-fluence regime.

Besides a laser fluence, the influence of a laser pulse overlap and a scan speed on $\mathrm{Al}_{2} \mathrm{O}_{3}$ removal rate in an air and water surroundings was investigated as shown in Fig. 2, using a 4.6-mJ pulse energy at 4-kHz repetition rate and a laser focal spot of $50 \mu \mathrm{m}$. Calculated pulse overlaps in percentage are also mentioned in Fig. 2. The term interpulse distance defines the distance separation from one pulse center to the 


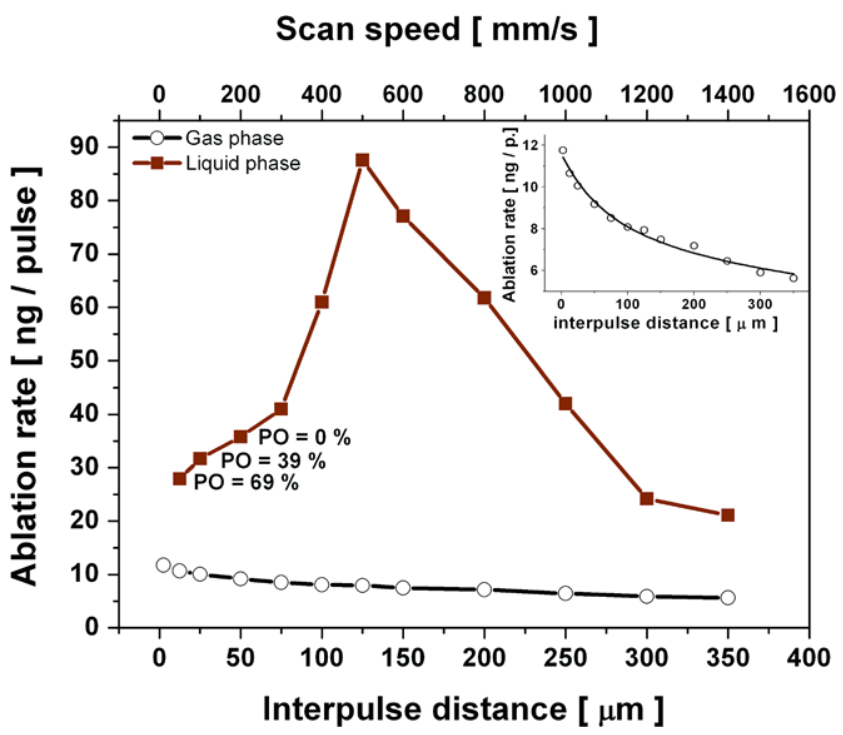

Fig. $2 \mathrm{Al}_{2} \mathrm{O}_{3}$ ablation rate as a function of an interpulse distance and a scan speed using $4.6 \mathrm{~mJ}$ of laser pulse energy at $4-\mathrm{kHz}$ repetition rate in an air and water environment. Calculated laser pulse overlaps (PO) are also demonstrated in percentage. Inset shows the zoom on the ablation rate curve obtained in an ambient air

center of the neighboring pulse. It was clearly observed that the interpulse distance has critical effect when ablating in liquid phase, while the gas-phase ablation was significantly less sensitive to this parameter. Ablating alumina in water revealed that the material removal rate increases constantly in the 10 to $125 \mu \mathrm{m}$ interpulse distance range resulting in 28 to $88 \mathrm{ng} /$ pulse $\left(2 \times 10^{-5} \mathrm{~g} / \mathrm{J}\right.$ of ablation efficiency), which is about $300 \%$ of enhancement compared to machining in pulse overlap mode. However, when the $125-\mu \mathrm{m}$ pulse separation is exceeded, this tendency is inverted and the ablation rate continuously decreases. We assume that two competitive effects tend to affect the ablation efficiency in a case of alumina. In the high overlap regime, the laser irradiation might be absorbed and/or scattered by previously ablated species and the previously generated cavitation bubble which prevent higher ablation efficiencies. Referring to the literature, such cavitation bubble lasts for about $300 \mu \mathrm{s}$ using Nd:YAG irradiation of $36 \mathrm{~J} / \mathrm{cm}^{2}$ of laser fluence [8]. Absorption and scattering effects decrease with increasing interpulse distance due to the limited size of the cavitation bubble and theoretically leads to a final saturation plateau in the ablation rate. Accordingly, we observed a rapid enhancement in the removal rate for an increasing scan speed to $500 \mathrm{~mm} / \mathrm{s}$. On the other hand, an increasing interpulse distance with equal laser parameters also results in significant variation in temperature gradient deposited into the lattice [13]. Heat of evaporation decreases with an increasing lattice temperature due to energy input supplied by the incident laser irradiation, and increasing temperature (heat accumulation) in the target lattice at constant density supports phase explosion [11]. We have to note that in the liquid flow

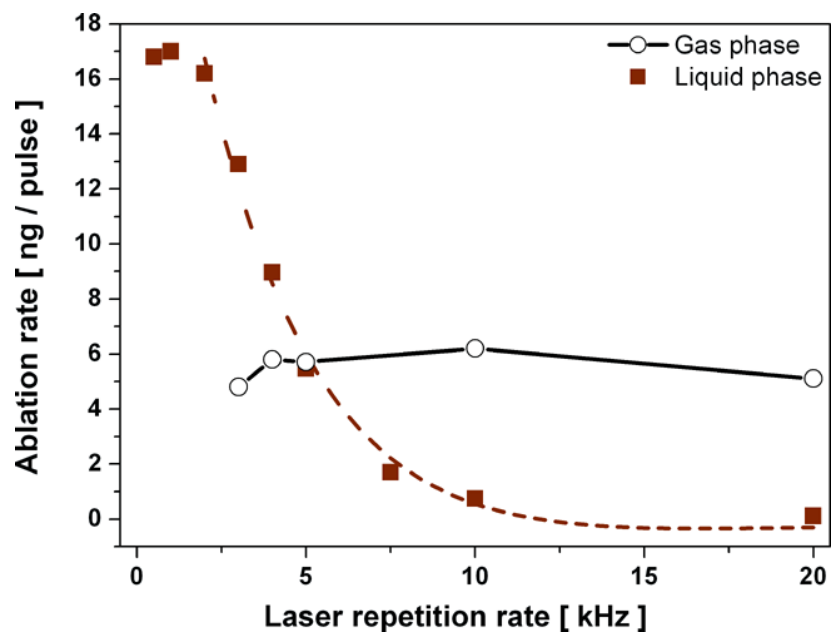

Fig. 3 Influence of the laser repetition rate on $\mathrm{Al}_{2} \mathrm{O}_{3}$ material removal rate with a $3.3-\mathrm{mJ}$ pulse energy and $125 \mu \mathrm{m}$ of interpulse distance. Presented fit is a monoexponential function

regime investigated the variation of a flow direction respect to a scan direction did not affect significantly the material removal rate. It is thus likely that such a low liquid flow speed could not affect significantly the spatial dynamics of the generated cavitation bubble. As the inset of Fig. 2 shows, $\mathrm{Al}_{2} \mathrm{O}_{3}$ ablation rate in an air is affected with an interpulse distance in an exponential manner proving the importance of a temperature gradient in the ceramic lattice. The significantly longer decay of the exponential confirms that in a liquid media, heat dissipation is accelerated by the surrounding.

This hypothesis of cavitation bubble and heat accumulation affected the laser ablation was confirmed by determining ablated rates in an air and water changing the laser repetition rate with a constant $125 \mu \mathrm{m}$ of interpulse distance and a $3.3 \mathrm{~mJ}$ of laser pulse energy, shown in Fig. 3. Investigations revealed a radical exponential drop of in the ablation rate when increasing repetition rate from 0.5 to $20 \mathrm{kHz}$ in a liquid phase reaching 17 and $0.27 \mathrm{ng} /$ pulse, respectively, while it was nearly constant $5.8 \mathrm{ng} / \mathrm{pulse}$ in a gas phase. Maximum ablation rate was gained at the specific laser repetition rate of $2 \mathrm{kHz}$ which is equal to $500 \mu$ s time delay between two laser pulses. This particular time delay is exactly in the order of the cavitation bubble lifetime [8]. It is thus likely that when the time between two laser pulses is long enough ( $2 \mathrm{kHz}$ or lower) the cavitation bubble collapses before the subsequent laser pulse impacts resulting in a saturation plateau in absolute removal rates. In a case of a gas phase ablation cavitation bubble formation is excluded, thus the ablation process is not influenced by the repetition rate.

Laser-generated particle size, size distribution, and morphology of water-dispersed ceramic nanoparticles were determined by transmission electron microscopy (TEM), 


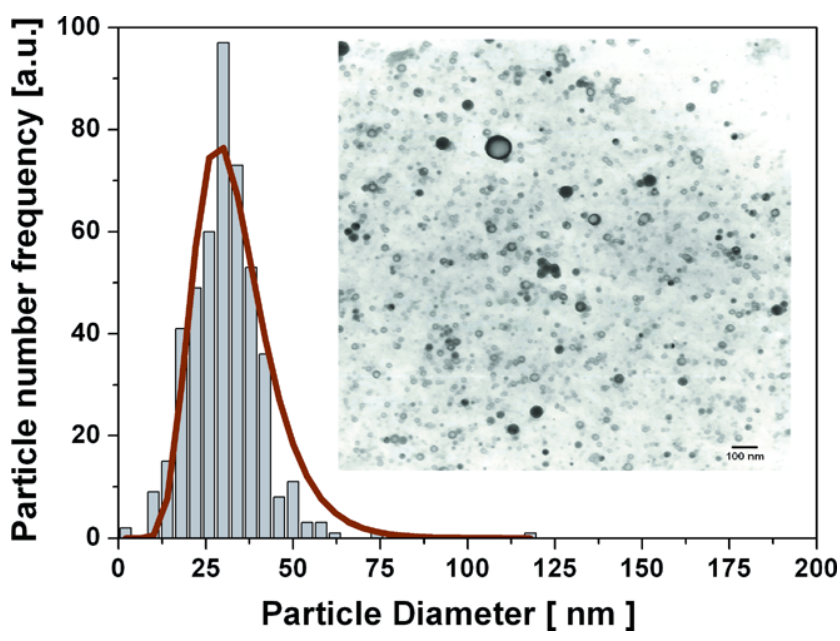

Fig. 4 TEM micrograph of $\mathrm{Al}_{2} \mathrm{O}_{3}$ nanoparticles and corresponding size distribution histogram prepared by the laser ablation in an aqueous environment using a $3.9-\mathrm{mJ}$ pulse energy at $4-\mathrm{kHz}$ repetition rate

shown in Fig. 4, revealing that high nanoparticle productivity in the order of $1 \mathrm{~g} / \mathrm{h}$ by optimal liquid laser ablation is associated with the generation of relatively small nanoparticles with narrow size distribution. Medium Feret nanoparticle diameter of $29 \mathrm{~nm}$ (Log normal fit) with 30-nm FWHM of dispersion was identified using a laser fluence of $202 \mathrm{~J} / \mathrm{cm}^{2}$ at 4-kHz repetition rate. TEM micrographs in Fig. 4 confirmed the presence of great majority of small spherical nanoparticles and only a few irregular-shaped (probably) cracked particles (not presented in Fig. 4) independently from the laser fluence used. Particle size and morphology have to be further investigated in detail as laser-induced fragmentation may also take place, reported for metal nanoparticles [14] which have a much higher absorption cross section than alumina.

\section{Conclusion}

In conclusion, nanosecond laser ablation of $\alpha-\mathrm{Al}_{2} \mathrm{O}_{3}$ in a liquid media yields a much higher ablation efficiency than that in a gas phase exceeding the ablation rate of $1.2 \mathrm{~g} / \mathrm{h}$ using a $4.6-\mathrm{mJ}$ focused pulse energy, a $4-\mathrm{kHz}$ repetition rate, and a specific interpulse distance of $125 \mu \mathrm{m}$ while generating nanoparticles of $29 \mathrm{~nm}$ in diameter with low dispersion of 30-nm FWHM. It is presumed that the ablation in a liquid phase is drastically influenced by the generated cavitation bubble and a heat deposited into the lattice, while in an ambient air, debris redeposition tends to influence ablation rate.

Acknowledgements The German Federal Ministry of Education and Research (project NANO-PART) and German Research Foundation (excellence cluster REBIRTH and project $\mathrm{CH}$ 179/9-1) are gratefully acknowledged for the financial support.

Open Access This article is distributed under the terms of the Creative Commons Attribution Noncommercial License which permits any noncommercial use, distribution, and reproduction in any medium, provided the original author(s) and source are credited.

\section{References}

1. F. Mafunè, J. Kohno, Y. Takeda, T. Kondow, J. Phys. Chem. B 104, $9111(2000)$

2. A.V. Simakin, V.V. Voronov, G.A. Shafeev, R. Brayner, F. BozonVerduraz, Chem. Phys. Lett. 348 (2001).

3. J.P. Sylvestre, A.V. Kabashin, E. Sacher, M. Meunier, Appl. Phys. A 80, 753 (2005)

4. J.A. Dahl, B.L.S. Maddux, J.E. Hutchison, Chem. Rev. 107, 2228 (2007)

5. N. Bärsch, A. Gatti, S. Barcikowski, J. Laser Micro/Nanoeng. 4, 66 (2007)

6. S. Zhu, Y.F. Lu, M.H. Hong, X.Y. Chen, J. Appl. Phys. 89, 2004 (2001)

7. S. Zhu, Y.F. Lu, M.H. Hong, Appl. Phys. Lett. 79, 1396 (2001)

8. T. Tsuji, Y. Tsuboi, Y. Kitamura, N. Tsuji, Appl. Surf. Sci. 229, 365 (2004)

9. T. Tsuji, Y. Okazaki, Y. Tsuboi, M. Tsuji, Jpn. J. Appl. Phys. 46, 1533 (2007)

10. S. Barcikowski, A.M. Menendez, B. Chichkov, M. Brikas, G. Raciukaitis, Appl. Phys. Lett. 91, 83113 (2007)

11. A. Miotello, R. Kelly, Appl. Phys. A 69, 67 (1999)

12. N.M. Bulgakova, A. Bulgakov, Appl. Phys. A 73, 199 (2001)

13. X. Wang, J.D. Shephard, F.C. Dear, D.P. Hand, J. Am. Ceram. Soc. 91, 391 (2008)

14. F. Mafuné, T. Kondow, Chem. Phys. Lett. 383, 343 (2004) 